UMN-TH-1901/00

TPI-MINN-00/23

hep-ph/0005146

May 2000

\title{
Solving the Hierarchy Problem in Two-Brane Cosmological Models
}

\author{
Panagiota Kanti, Keith A. Olive and Maxim Pospelov \\ Theoretical Physics Institute, School of Physics and Astronomy, \\ University of Minnesota, Minneapolis, MN 55455, USA
}

\begin{abstract}
We analyze cosmological solutions in the class of two-brane models with arbitrary tensions which contain matter with general equations of state. We show that the mass hierarchy between the two branes is determined by the ratio of the lapse functions evaluated on the branes. This ratio can be sufficiently small without fine-tuning the brane separation, once the transverse dimension is stabilized. For suitably large interbrane separations, both brane tensions are positive. We also find that the cosmological evolution obeys the standard four-dimensional Friedman equation up to small corrections.
\end{abstract}




\section{Introduction}

Physical consequences of extra space-like dimensions have been the subject of intensive theoretical investigations over the past two years. In general, the presence of new dimensions can change our interpretation of the fundamental parameters in the theory. Four-dimensional constants, such as Plank mass, electroweak scale, coupling constants and so on, are, in fact, functions of the volume of the extra dimensions. This dependence is particularly interesting in theories where gravity and the rest of the Standard Model fields "live" in different numbers of dimensions. This can be realized in the brane-world scenario, in which Standard Model particles are confined to a $3+1$ space-time dimensional manifold, embedded into a higher-dimensional space. A very well known stringy prototype of these constructions [1], uses a relatively large size of the transverse 11-th dimension in supergravity to reconcile effective Plank scale and GUT/string scale. In such a construction the 4D-Planck scale, $M_{P}$, is derived from the fundamental scale, $M_{11}$, using $M_{P}^{2}=M_{11}^{3} R_{11}$ having assumed that the 6-space volume, $V_{6} \sim M_{11}^{-6}$. For $R_{11} \gg M_{G U T}$, we can obtain, $M_{G U T} \sim M_{11} \ll M_{P}$, thus relaxing the hierarchy problem of the GUT and string scales.

Interest in such models, driven by particle phenomenology and cosmology, was further amplified when the brane world idea was used to lower the fundamental gravitational scale, bringing it close to the weak scale [2] in an attempt to resolve the hierarchy problem between the weak and Planck scales. Indeed, as it was shown in [2], due to a possibly very large volume of extra dimensions felt only by gravity, the fundamental scale could be much lower than the one inferred from measuring the Newtonian force in four dimensions. This opens up a number of interesting phenomenological consequences, including the possibility of observing higher-dimensional gravitational interactions in collider experiments. An alternative to solve the weak scale hierarchy problem was proposed by Randall and Sundrum [3]. Rather than requiring a large extra dimension, one simply constructs an exponentially large ratio of the two length scales on two different branes, which results from an "exponentially decaying" scale factor (or warp factor) in AdS geometry. For other attempts at solving the hierarchy problem with extra dimensions see [4].

The study of the cosmological evolution in this type of model has undergone several interesting turns. It was shown in [5, 6, 7] that the Hubble parameter on the brane is proportional to the brane tension, rather than to the square root of it, as one would expect from pure low-energy four-dimensional reasoning. Two-brane models of this type have additional problems since the matter densities $\rho_{1}, \rho_{2}$ and pressures $p_{1}$ and $p_{2}$ need to be correlated in order to achieve a consistent solution (in fact one would derive that $\rho_{2} \simeq-\rho_{1}$ which lacks a consistent interpretation). This also leads to the correlation between the states of matter on the two different branes. These problems with cosmology are indicative that some crucial ingredients are missing in the brane models which would allow a smooth 
transition to "normal" four-dimensional physics. It was conjectured in 8] and further proven in [9, 10] that the missing element in brane models is the stabilization of the extra dimension.

It was shown in Refs. [8, 10] that single-brane models with a compactified extra dimension lead to standard cosmology, irrespective of the nature of the precise mechanism responsible for the stabilization of the transverse direction. In technical terms, stabilization provides a bulk value for the transverse pressure-like component of the stress-energy tensor, proportional to the trace of the stress-energy tensor of the brane. By including the bulk component of energy-momentum tensor $\left(T_{55}\right)$, one not only recovers the standard form of the Friedmann equation, but also relieves the necessity of the (anti) correlation of the energy density on the brane allowing for the construction of realistic two- and one-brane models. In [10], it was shown that $T_{55}$ is automatically generated as a back-reaction to matter on the brane and induces a shift (proportional to $T_{\mu}^{\mu}$ ) in the minimum of the dilaton potential. However, in such models with matter, the warp factor is insignificant (it is proportional to $\left.\rho / M_{P}^{4}\right)$ and we lose the potential solution to the hierarchy problem.

In our subsequent paper [11], we generalized this construction and found a static solution for the case of two positive tension branes, assuming the same stabilization mechanism. An interesting feature of this construction, noted in [11], is the possibility of the exponentially large ratio between the two scales on two different branes, which allows to address the gauge hierarchy problem a là Randall and Sundrum (RS) [3], and at the same time avoid the presence of the negative tension brane. Two positive tension branes are allowed in this context because the solution to the warp factor is cosh-like rather than a pure exponential. Even though the negative tension branes are plausible in string theory, their existence in the real physical world is questionable. Indeed, these branes may turn out to be unstable, once the supersymmetry is broken, and to the best of our knowledge this question has not been properly studied so far. In contrast, positive tension branes are ubiquitous, and occur not only in string theory, but also in an ordinary field theories where they can simply be interpreted as domain walls, domain wall junctions, etc.

We believe that this idea [1] deserves further consideration, and in this paper we take it one step further, introducing matter density and pressure on the two branes in order to obtain consistent cosmological solutions. In accordance with what we learned in the case of the single-brane models, this expansion is exactly of the Friedmann form, provided that the transverse dimension is stabilized. At the same time, there is no correlation between $\rho_{1}$, $\rho_{2}, p_{1}$ and $p_{2}$, and no restriction on the equations of state. We obtain general solutions for arbitrary brane tensions and we further demonstrate that it is possible to recover a solution to the hierarchy problem for negative or positive values of the observable brane tension. As a result, the general system of two branes discussed in this paper gives rise to cosmological solutions that include, as special cases, both the positive-negative brane combination of 
the RS model [3] and the more natural case of the two positive tension branes [11]. We find that the system is characterized by the presence of three parameters: the ratio of the self-energy over the bulk cosmological constant of each brane and the inter-brane distance. Demanding the cancellation of the effective cosmological constant, we place one constraint on a combination of these parameters while the resolution of the hierarchy problem fixes one more parameter. We keep the inter-brane distance, $L$, as the free parameter and we demonstrate that suitably small values of the warp factor, which we show to be directly related to the ratio $M_{P} / M_{W}$, is achieved for any value of $L$. In other words, no other fine tuning is necessary to obtain the necessary warp factor which we show to be directly related to the ratio of the lapse functions on the two branes.

As indicated above, we find a normal Friedmann expansion on the visible brane and show that in fact both the visible and hidden branes expand with the same expansion rate. Upon rescaling the matter density on the visible brane, we further show that the deduced Plank scale is the same on both branes. Although the scale factors and lapse functions change in time leading to a time dependent ratio of weak scale and four-dimensional Plank constant, we show that the residual change in $M_{W} / M_{P}$ is minute, suppressed by the ratios of $\rho_{i} / M_{P}^{4}$, and thus representing no danger to this model.

This paper is organized as follows. In section 2, we introduce the ansatz for the metric, stress-energy tensor and present the five-dimensional Einstein's equations. In the next section, we derive the solution for the spatial scale factor, in the bulk, consistent with the brane boundary conditions, and show that the cosmological expansion rate on the two branes is of the form of the standard four-dimensional Friedmann equation, once the interbrane distance is stabilized. In section 4, we consider the solution to the hierarchy problem with branes with arbitrary tensions, and define the necessary conditions for this to happen. We demonstrate that the hierarchy problem can be resolved for arbitrary values of the interbrane separation, by appropriately choosing the value of the observable brane tension, with the RS choice of parameters arising only as a special case. We draw our conclusions in section 5 .

\section{The Field Equations}

The starting point of our analysis will be the assumption of the existence of an extra dimension, denoted by the coordinate $y$, in addition to the usual four coordinates, $\left\{t, x^{i}\right\}$, of the 4-dimensional spacetime. We consider the following ansatz for the line-element of the 5-dimensional manifold

$$
d s^{2}=-n^{2}(t, y) d t^{2}+a^{2}(t, y) \delta_{i j} d x^{i} d x^{j}+b^{2}(t, y) d y^{2}
$$


where $a(t, y)$ and $b(t, y)$ are the scale factors of the 4-dimensional spacetime (which we assume is spatially flat, i.e., ${ }^{3} k=0$ ) and the extra dimension, respectively, and $n(t, y)$ the lapse function that defines the time variable. Motivated by M-theory [1], we assume that the extra dimension has the topology of an orbifold, i.e. is a circle where the discrete $Z_{2}$ symmetry, $y \leftrightarrow-y$, has been imposed. Two 3-branes of zero thickness located at the orbifold's fixed points, $y=0$ and $y=L$, set the size of the extra dimension to be equal to $2 b L$. The inter-brane distance is assumed to remain fixed due to a stabilization mechanism which ensures that $\dot{b}=b^{\prime}=0$. The coordinate $y$ is scaled so that we may set, for simplicity, $b=1$. Note that, throughout the paper, dots and primes denote differentiation with respect to time and $y$, respectively. For other approaches to brane-world cosmologies, see [12].

We now turn to the 5-dimensional action that describes the coupling of the matter content of the universe with gravity. We assume that all the usual matter fields are localized on the two 3-branes, at $y=0$ and $y=L$, that play the role of a hidden and observable universe, respectively. The two branes are characterized by self-energies $\Lambda_{1}$ and $\Lambda_{2}$, while a non-vanishing cosmological constant $\Lambda_{B}$ exists in the bulk. Under the above assumption, the action takes the form

$$
S=-\int d^{4} x d y \sqrt{-\hat{g}}\left\{\frac{M_{5}^{3}}{16 \pi} \hat{R}+\Lambda_{B}+\Lambda_{1} \delta(y)+\Lambda_{2} \delta(y-L)+\hat{\mathcal{L}}_{o}\right\} .
$$

In the above, $\hat{\mathcal{L}}_{o}$ represents all possible contributions to the action which are not strictly gravitational, $M_{5}$ is the fundamental 5-dimensional Planck mass and the hat denotes 5dimensional quantities.

Einstein's equations, with the above spacetime background (2.1) and the assumption that $\dot{b}=b^{\prime}=0$, take the simplified form (for the full version of these equations see e.g. Refs. [5, [7])

$$
\begin{aligned}
& \hat{G}_{00}=3\left\{\left(\frac{\dot{a}}{a}\right)^{2}-n^{2}\left[\frac{a^{\prime \prime}}{a}+\left(\frac{a^{\prime}}{a}\right)^{2}\right]\right\}=\hat{\kappa}^{2} \hat{T}_{00} \\
& \hat{G}_{i i}=a^{2}\left\{\frac{a^{\prime}}{a}\left(\frac{a^{\prime}}{a}+2 \frac{n^{\prime}}{n}\right)+2 \frac{a^{\prime \prime}}{a}+\frac{n^{\prime \prime}}{n}\right\}+\frac{a^{2}}{n^{2}}\left\{\frac{\dot{a}}{a}\left(-\frac{\dot{a}}{a}+2 \frac{\dot{n}}{n}\right)-2 \frac{\ddot{a}}{a}\right\}=\hat{\kappa}^{2} \hat{T}_{i i}, \\
& \hat{G}_{05}=3\left(\frac{n^{\prime}}{n} \frac{\dot{a}}{a}-\frac{\dot{a}^{\prime}}{a}\right)=0 \\
& \hat{G}_{55}=3\left\{\frac{a^{\prime}}{a}\left(\frac{a^{\prime}}{a}+\frac{n^{\prime}}{n}\right)-\frac{1}{n^{2}}\left[\frac{\dot{a}}{a}\left(\frac{\dot{a}}{a}-\frac{\dot{n}}{n}\right)+\frac{\ddot{a}}{a}\right]\right\}=\hat{\kappa}^{2} \hat{T}_{55},
\end{aligned}
$$

where $\hat{\kappa}^{2}=8 \pi \hat{G}=8 \pi / M_{5}^{3}$. In the above, $\hat{T}_{M N}$ is the total 5-dimensional energy-momentum tensor which can be decomposed in terms of the bulk and brane contributions as follows

$$
\hat{T}_{M N}=\hat{T}_{M N}^{(B)}+\hat{T}_{M N}^{(1)}+\hat{T}_{M N}^{(2)}
$$


where

$$
\begin{aligned}
& \hat{T}_{(B) N}^{M}=\operatorname{diag}\left(-\Lambda_{B},-\Lambda_{B},-\Lambda_{B},-\Lambda_{B}, \hat{T}_{(B) 5}^{5}\right), \\
& \hat{T}_{(1) N}^{M}=\operatorname{diag}\left[\frac{\delta(y)}{b}\left(-\rho_{1}-\Lambda_{1}, p_{1}-\Lambda_{1}, p_{1}-\Lambda_{1}, p_{1}-\Lambda_{1}\right), 0\right], \\
& \hat{T}_{(2) N}^{M}=\operatorname{diag}\left[\frac{\delta(y-L)}{b}\left(-\rho_{2}-\Lambda_{2}, p_{2}-\Lambda_{2}, p_{2}-\Lambda_{2}, p_{2}-\Lambda_{2}\right), 0\right] .
\end{aligned}
$$

Note that we allow for a non-vanishing value of the (55)-component of the energy-momentum tensor in the bulk that includes, in addition to the contribution due to the bulk cosmological constant, a part proportional to the trace of the energy-momentum tensor on the brane. As it has been shown [8, 9, 10], this component results from a 5-dimensional stabilization mechanism which is responsible for keeping the inter-brane distance, and thus the size of the extra dimension, fixed. The presence of a stabilizing potential for the radion field in the framework of the 5-dimensional, fundamental theory leads to a $(t, y)$ dependent value for $\hat{T}_{(B) 5}^{5}$ distinctly different from $-\Lambda_{B}$. In particular, it can be shown that $\hat{T}_{(B) 5}^{5}+\Lambda_{B}$ is (to leading order in $\rho$ ) proportional to the trace $T_{\mu}^{\mu}$ on the brane. For later use, we display here the relations that follow from the conservation of the energy-momentum tensor, $D_{M} \hat{T}^{M}{ }_{N}=0$, on the branes

$$
\frac{d \rho_{1}}{d t}+3\left(\rho_{1}+p_{1}\right) \frac{\dot{a}_{0}}{a_{0}}=0, \quad \frac{d \rho_{2}}{d t}+3\left(\rho_{2}+p_{2}\right) \frac{\dot{a}_{L}}{a_{L}}=0
$$

and in the bulk

$$
\left(\hat{T}_{(B) 5}^{5}\right)^{\prime}+\hat{T}_{(B) 5}^{5}\left(\frac{n^{\prime}}{n}+3 \frac{a^{\prime}}{a}\right)+\Lambda_{B}\left(\frac{n^{\prime}}{n}+3 \frac{a^{\prime}}{a}\right)=0
$$

Here, we closely follow the analysis of Ref. [10] which we extend to the case of two branes with arbitrary self-energies $\Lambda_{i}$ 's. Our subsequent analysis will be greatly simplified by the fact that the (05)-component of Einstein's equations (2.5) can be easily integrated to give the result

$$
n(t, y)=\lambda(t) \dot{a}(t, y) .
$$

Using the normalization $n(t, y=0)=1$, the arbitrary function of time $\lambda(t)$ turns out to be $\lambda(t)=1 / \dot{a}_{0}$ in terms of which, as we will shortly see, the Hubble parameter on both branes can be easily expressed.

\section{Cosmological Evolution}

In this section, we focus on the general solution for the scale factor $a(t, y)$ which is consistent with the aforementioned energy distribution in the 5-dimensional universe and the boundary 
conditions that the two, infinitely thin, brane-universes introduce in the theory. We will also determine the form of the generalized Friedmann equations that the boundary quantities $a_{0}$ and $a_{L}$ satisfy and we will investigate the conditions necessary for the restoration of the usual Friedmann equation on the two branes, through the vanishing of the effective cosmological constant, as well as for the successful stabilization of the extra dimension.

We note that by using the relation (2.11), the (00)-component of Einstein's equations (2.3), in the bulk, reduces to an ordinary second-order differential equation for $a(t, y)$ with respect to $y$. As a result, we can easily find the general solution for the scale factor, in the bulk, which, for $\Lambda_{B}<0$, has the form

$$
a^{2}(t, y)=d_{1}(t) \cosh (A|y|)+d_{2}(t) \sinh (A|y|)-\frac{B^{2}(t)}{A^{2}}
$$

where

$$
A^{2}=\frac{2 \hat{\kappa}^{2}}{3}\left|\Lambda_{B}\right|, \quad B^{2}(t)=\frac{2}{\lambda^{2}(t)},
$$

and where $d_{1}$ and $d_{2}$ are two functions of time which will be shortly determined. The above solution, derived in the bulk, needs to be smoothly connected to its boundary values $a_{0}$ and $a_{L}$. We can write

$$
(\mathbf{A}): \quad a^{2}(t, 0)=a_{0}^{2}(t), \quad a^{2}(t, L)=a_{L}^{2}(t) .
$$

The first of these conditions leads to the determination of the unknown function $d_{1}(t)$,

$$
d_{1}(t)=a_{0}^{2}(t)+\frac{B^{2}(t)}{A^{2}}
$$

while the second leads to the relation between the two boundary values $a_{0}$ and $a_{L}$

$$
a_{L}^{2}(t)=d_{1}(t) \cosh (A L)+d_{2}(t) \sinh (A L)-\frac{B^{2}(t)}{A^{2}} .
$$

Two more sets of boundary conditions need to be satisfied by the general solution (3.12). These conditions follow from the inhomogeneity in the distribution of matter in the universe and involve the jumps in the first derivatives of $a$ and $n$ across the two branes. They have the form

$$
\begin{aligned}
& \text { (B) : } \quad \frac{\left[a^{\prime}\right]_{0}}{a_{0}}=-\frac{\hat{\kappa}^{2}}{3}\left(\rho_{1}+\Lambda_{1}\right), \quad \frac{\left[a^{\prime}\right]_{L}}{a_{L}}=-\frac{\hat{\kappa}^{2}}{3}\left(\rho_{2}+\Lambda_{2}\right), \\
& (\mathbf{C}): \quad \frac{\left[n^{\prime}\right]_{0}}{n_{0}}=\frac{\hat{\kappa}^{2}}{3}\left(3 p_{1}+2 \rho_{1}-\Lambda_{1}\right), \quad \frac{\left[n^{\prime}\right]_{L}}{n_{L}}=\frac{\hat{\kappa}^{2}}{3}\left(3 p_{2}+2 \rho_{2}-\Lambda_{2}\right) .
\end{aligned}
$$

We first focus on set $(\mathbf{B})$ : the condition at $y=0$ determines the remaining arbitrary function $d_{2}(t)$ to be

$$
d_{2}(t)=-\frac{\hat{\kappa}^{2}}{3 A} a_{0}^{2}\left(\rho_{1}+\Lambda_{1}\right),
$$


while the same condition at $y=L$, combined with the relation (3.16), leads to two extremely interesting results: the Friedmann equation at $y=0$,

$$
\left(\frac{\dot{a}_{0}}{a_{0}}\right)^{2}=\frac{\hat{\kappa}^{2}\left|\Lambda_{B}\right|}{3} \frac{\left[-\Lambda_{R S}+\frac{\left(\rho_{1}+\Lambda_{1}+\rho_{2}+\Lambda_{2}\right)}{\tanh (A L)}-\frac{\left(\rho_{1}+\Lambda_{1}\right)\left(\rho_{2}+\Lambda_{2}\right)}{\Lambda_{R S}}\right]}{\Lambda_{R S}-\left(\rho_{2}+\Lambda_{2}\right) \tanh \left(\frac{A L}{2}\right)},
$$

and the ratio of the scale factors on the two branes

$$
\frac{a_{L}^{2}}{a_{0}^{2}}=\frac{\Lambda_{R S}-\left(\rho_{1}+\Lambda_{1}\right) \tanh \left(\frac{A L}{2}\right)}{\Lambda_{R S}-\left(\rho_{2}+\Lambda_{2}\right) \tanh \left(\frac{A L}{2}\right)}
$$

expressed in terms of $\Lambda_{R S} \equiv \sqrt{6\left|\Lambda_{B}\right| / \hat{\kappa}^{2}}, \Lambda_{i}, \rho_{i}$ and $L$. The above form of the Friedmann equation has also been derived in Ref. [13] while some of the expressions presented in this section have been determined, in the special case $\Lambda_{1}=-\Lambda_{2}=\Lambda_{R S}$, in Ref. [14]. As in the case of static solutions [11], the ratio of the scale factors on the two branes is given in terms of the 'detuning' of the total energy densities of the branes from the limiting value $\Lambda_{R S}$. Differentiating the above expression with respect to time, we are able to derive the form of the Hubble parameter on the second brane, which is

$$
\left(\frac{\dot{a}_{L}}{a_{L}}\right)=\left(\frac{\dot{a}_{0}}{a_{0}}\right)\left[\frac{\Lambda_{R S}-\left(\rho_{2}+\Lambda_{2}\right) \tanh \left(\frac{A L}{2}\right)}{\Lambda_{R S}-\left(\rho_{1}+\Lambda_{1}\right) \tanh \left(\frac{A L}{2}\right)}\right]\left[\frac{2 \Lambda_{R S}-\left(2 \Lambda_{1}-\rho_{1}-3 p_{1}\right) \tanh \left(\frac{A L}{2}\right)}{2 \Lambda_{R S}-\left(2 \Lambda_{2}-\rho_{2}-3 p_{2}\right) \tanh \left(\frac{A L}{2}\right)}\right] .
$$

Finally, let us note that the set $(\mathbf{C})$ of boundary conditions involving the jumps of the lapse function $n$ across the two branes must also be satisfied. By using the fact that $n(t, y)=\dot{a}(t, y) / \dot{a}_{0}(t)$, differentiating with respect to $y$ and substituting in eqs. (3.18), we may see, after some algebra, that the jump conditions on both boundaries are identically satisfied. The above result reveals the absence of any correlation between the equations of state on the two brane-universes. This feature was also pointed out in Ref. [14], in the special case $\Lambda_{1}=-\Lambda_{2}=\Lambda_{R S}$, however, it turns out to be valid even in the case where the self-energies of the branes and the bulk cosmological constant are completely arbitrary and uncorrelated.

We now turn to the conditions that we need to impose in order to recover the usual Friedmann equation on both branes. The most obvious is the vanishing of the effective cosmological constant. From eq. (3.20), this translates to

$$
\Lambda_{e f f} \sim \Lambda_{R S}\left(-1-\alpha \beta+\frac{\alpha+\beta}{\tanh (A L)}\right)=0
$$

where we have defined

$$
\alpha \equiv \frac{\Lambda_{1}}{\Lambda_{R S}}, \quad \beta \equiv \frac{\Lambda_{2}}{\Lambda_{R S}} .
$$

The condition (3.23) involves three parameters, $\alpha, \beta$ and $L$ which, until now, were completely uncorrelated. The condition of the vanishing of $\Lambda_{e f f}$ renders one of them a dependent 
parameter, thus, reducing the number of independent parameters to two. Note that there is a special choice for the parameters $\alpha$ and $\beta$ that eliminates $\Lambda_{e f f}$, for every value of the inter-brane distance $L$. This is $\alpha=-\beta= \pm 1$ and corresponds to the Randall-Sundrum choice [3]. For every other combination of $\alpha$ and $\beta$, the distance between the two branes has to be carefully chosen in order to ensure that $\Lambda_{\text {eff }}=0$.

Although the vanishing of the effective cosmological constant has simplified the Friedmann-like equation at $y=0$, eq. (3.20), it has not completely restored its usual form. The existence of two branes with non-vanishing energy densities, $\rho_{i}$, leads to the appearance of both $\rho_{1}$ and $\rho_{2}$, in the equations that govern the cosmological evolution of each brane. Besides terms linear in $\rho_{1}$ and $\rho_{2}$, the resulting cosmological equations will be corrected by higher powers of energy densities, i.e. $\rho_{1}^{2}, \rho_{2}^{2}, \rho_{1} \rho_{2}$, and so on. Exact numerical coefficients in front of these terms are not known, as they depend on subtle details of radius stabilization [10]. However, for a natural choice of parameters, these terms can easily be shown to be of secondary importance due to their extremely small magnitude. If we assume that $\Lambda_{R S} \sim M_{P}^{4}$ while $\rho_{i} \lesssim \rho_{c} \simeq 10^{-123} M_{P}^{4}$ (as dictated by the observable expansion rate of our $4 \mathrm{D}$ universe), and expand eq. (3.20) keeping only terms linear in $\rho_{i} / \Lambda_{R S}$, we obtain the result

$$
\left(\frac{\dot{a}_{0}}{a_{0}}\right)^{2}=\frac{\hat{\kappa}^{2}\left|\Lambda_{B}\right|}{3 \Lambda_{R S} \tanh (A L)}\left\{\frac{1-\beta \tanh (A L)}{1-\beta \tanh \left(\frac{A L}{2}\right)} \rho_{1}+\frac{1-\alpha \tanh (A L)}{1-\beta \tanh \left(\frac{A L}{2}\right)} \rho_{2}\right\} .
$$

We could also use the condition of the vanishing of $\Lambda_{\text {eff }}$ in order to eliminate one of the three parameters $\alpha, \beta$ and $L$. We have chosen to eliminate $\alpha$, using (3.23). The final form of the Friedmann equation, at $y=0$, then takes the form

$$
\left(\frac{\dot{a}_{0}}{a_{0}}\right)^{2}=\frac{\hat{\kappa}^{2}\left|\Lambda_{B}\right|}{3 \Lambda_{R S} \tanh (A L)} \frac{1-\beta \tanh (A L)}{1-\beta \tanh \left(\frac{A L}{2}\right)}\left\{\rho_{1}+\frac{\rho_{2}}{[\cosh (A L)-\beta \sinh (A L)]^{2}}\right\} .
$$

Before commenting on the above result, we should point out that the Friedmann equation on the observable brane, at $y=L$, has exactly the above form, in the same linear approximation. We can easily see, from eq. (3.22), that by ignoring terms of $\mathcal{O}\left(\rho_{i} / \Lambda_{R S}\right)^{2}$ or $\mathcal{O}\left(\rho_{i} \rho_{j} / \Lambda_{R S}^{2}\right)$, the two Hubble parameters become identical. In eq. (3.26) we would restore the normal 4-dimensional Hubble expansion law if we define Newton's constant as

$$
\kappa^{2}=\frac{\hat{\kappa}^{2}\left|\Lambda_{B}\right|}{\Lambda_{R S} \tanh (A L)} \frac{1-\beta \tanh (A L)}{1-\beta \tanh \left(\frac{A L}{2}\right)} .
$$

The square of the Hubble parameter is now proportional to the total energy density of the universe, which contains contributions from both branes. Note that the constant coefficient in front of the energy density of our brane-universe, $\rho_{2}$, is not unity (this was first pointed out in Ref. [9]). As was discussed in [14, 15], the observable energy density on our universe must be redefined in such a way as to absorb this coefficient. In the next section we will 
see that this redefinition is a result of the conformal transformation necessary to solve the hierarchy problem.

Finally, we need to address the problem of the stabilization of the extra dimension and determine the constraints on the energy distribution of the system that makes this stabilization possible. As we mentioned before and demonstrated in earlier works [8, 10], the stabilization mechanism responsible for keeping fixed the inter-brane distance manifests itself through the existence of a non-vanishing (55)-component of the energy-momentum tensor. The (55)-component of Einstein's equations, that we have ignored so far, will serve to determine the expression of this extra component. For the general solution (3.12), eq. (2.6) takes the form

$$
\frac{d}{d t}\left[\frac{A^{2}}{4}\left(d_{2}^{2}-d_{1}^{2}\right)+\frac{1}{A^{2} \lambda^{4}}\right]=\frac{2 \hat{\kappa}^{2}}{3} a^{3} \dot{a}\left(\hat{T}_{(B) 5}^{5}-\left|\Lambda_{B}\right|\right) .
$$

Substituting $d_{1}, d_{2}$ and $\lambda$ from eqs. (3.15), (3.19) and (3.20), respectively, we obtain the result

$$
\begin{aligned}
\hat{T}_{(B) 5}^{5} & =\left|\Lambda_{B}\right|+\frac{a_{0}^{3} \hat{\kappa}^{2}}{12 n a^{3}}\left\{\left(\rho_{1}+\Lambda_{1}\right)\left(2 \Lambda_{1}-\rho_{1}-3 p_{1}\right)+\Lambda_{R S}\left[2 \Lambda_{R S}-\frac{\left(4 \Lambda_{1}+\rho_{1}-3 p_{1}\right)}{\tanh (A L)}\right]\right. \\
& \left.-\frac{\Lambda_{R S}}{\sinh (A L)} \frac{a_{L}}{a_{0}}\left[2 \frac{a_{L}}{a_{0}}\left(\rho_{2}+\Lambda_{2}\right)+n_{L}\left(2 \Lambda_{2}-3 p_{2}-\rho_{2}\right)\right]\right\}
\end{aligned}
$$

and to linear order in $\rho_{i}$

$$
\begin{aligned}
\hat{T}_{(B) 5}^{5} & =\left|\Lambda_{B}\right|+\frac{a_{0}^{3}\left|\Lambda_{B}\right|}{2 n a^{3}}\left\{\frac{2\left(\beta^{2}-1\right)}{\cosh ^{2}(A L)[1-\beta \tanh (A L)]^{2}}\right. \\
& \left.-\frac{\left(\rho_{1}-3 p_{1}\right)\left[1-\beta \tanh \left(\frac{A L}{2}\right)\right]+\left(\rho_{2}-3 p_{2}\right)\left[1+\beta \tanh \left(\frac{A L}{2}\right)\right]}{\Lambda_{R S} \sinh (A L) \cosh (A L)[1-\beta \tanh (A L)]}\right\} .
\end{aligned}
$$

Note that this expression already assumes the vanishing of the cosmological constant (3.23). We may easily check that the expression (3.29), as well as the linearized version above, with $\hat{T}_{(B) 5}^{5} \sim w(t) / n(t, y) a^{3}(t, y)$, where $w(t)$ an arbitrary function of time, is consistent with the fifth component of the equation for the conservation of energy (2.10). In addition, as we expected, the coefficient $w$ is proportional to the trace of the energy momentum tensor on the branes [10, 16]. Note also that we neglect any small changes in $b$, due to changes in $\hat{T}_{(B) 5}^{5}$ or $\rho_{i}$, since these are proportional to $\rho_{i}^{3 / 2}$ and we consistently ignore terms of this order throughout our analysis.

\section{Resolution of the Hierarchy Problem}

After having stabilized the extra dimension and derived the Friedmann equations that govern the cosmological evolution of each brane, we now focus our attention on the observable 
brane-universe. Our goal will be to determine the correct definition of mass scales, $\tilde{m}$, and energy density, $\tilde{\rho}_{2}$, as measured by a 4D observer in a FRW background, in terms of the corresponding quantities, $m$ and $\rho_{2}$, defined on our 4-brane which, however, is part of a curved, non-FRW spacetime. (In this context, by FRW we refer to a metric with lapse function $n=1$, with only a time dependent spatial scale factor.) To this end, we consider the theory of a scalar field, $\Phi$, confined on our brane-universe which is embedded in a $5 \mathrm{D}$ spacetime background. Such a toy model was also considered in Ref. [3], however, here, we extend it to the case of a non-static spacetime background of the form (2.1). We consider the following action

$$
\begin{aligned}
S & =\int d^{4} x d y \sqrt{\hat{g}}\left[\hat{g}^{\mu \nu} \partial_{\mu} \Phi \partial_{\nu} \Phi+\lambda\left(\Phi^{2}-v_{0}^{2}\right)^{2}\right] \delta(y-L) \\
& =\int d^{4} x \sqrt{g_{L}}\left[g_{L}^{\mu \nu} \partial_{\mu} \Phi \partial_{\nu} \Phi+\lambda\left(\Phi^{2}-v_{0}^{2}\right)^{2}\right]
\end{aligned}
$$

where $g_{L \mu \nu}=\hat{g}_{\mu \nu}(y=L)$, is the induced metric tensor on our brane. Using the ansatz (2.1), the above action takes the form

$$
\begin{aligned}
S & =\int d^{4} x\left(a_{L}^{3} n_{L}\right)\left\{\left[-\frac{1}{n_{L}^{2}} \dot{\Phi}^{2}+\frac{1}{a_{L}^{2}}(\nabla \Phi)^{2}\right]+\lambda\left(\Phi^{2}-v_{0}^{2}\right)^{2}\right\} \\
& =\int d^{4} x \sqrt{\tilde{g}} n_{L}^{4}\left[\frac{1}{n_{L}^{2}} \tilde{g}^{\mu \nu} \partial_{\mu} \Phi \partial_{\nu} \Phi+\lambda\left(\Phi^{2}-v_{0}^{2}\right)^{2}\right]
\end{aligned}
$$

where a conformal transformation

$$
g_{L \mu \nu} \rightarrow \tilde{g}_{\mu \nu}=\frac{1}{n_{L}^{2}} g_{L \mu \nu}
$$

has been performed that restores the FRW character of the 4D spacetime and brings the corresponding line-element to the form

$$
d \tilde{s}^{2}=-d t^{2}+\tilde{a}^{2}(t) \delta_{i j} d x^{i} d x^{j}
$$

where $\tilde{a}(t) \equiv a_{L}(t) / n_{L}(t)$ is the observed scale factor of our universe. The last step we need to make involves the rescaling of the scalar field in order to absorb the gravitational coefficients that remain in the action. By setting $\phi \rightarrow \tilde{\phi}=n_{L} \phi$, we arrive at

$$
S=\int d^{4} x \sqrt{\tilde{g}}\left[\tilde{g}^{\mu \nu} \partial_{\mu} \tilde{\Phi} \partial_{\nu} \tilde{\Phi}+\lambda\left(\tilde{\Phi}^{2}-\tilde{v}_{0}^{2}\right)^{2}\right]
$$

The $v e v v_{0}$ of the scalar field has also been rescaled, $\tilde{v}_{0}=n_{L} v_{0}$, and as a result, the relation between any mass scale, $\tilde{m}$, defined in terms of the theory (4.5) and the corresponding one, $m$, defined in terms of (4.1), has the form

$$
\tilde{m}=n_{L}(t) m
$$


We may, thus, conclude that the rescaling coefficient that relates any effective mass scale with the corresponding fundamental one, in a general spacetime background, is the lapse function of the original 5D line-element evaluated at the location of the brane. In the case of the RS model, $n_{L}=e^{-b k L}$ and thus any mass scale $\tilde{m}$ is exponentially suppressed compared to $m$. Due to the fact that $n_{L}=a_{L}$, the induced line-element, in their case, was static since $\tilde{a}(t)=a_{L} / n_{L}=1$. In our case, however, the presence of the energy density $\rho_{2}$ inevitably leads to the expansion of our brane and to the time-dependence of the rescaling factor $n_{L}$. In Ref. [14], it was argued that such a rescaling is not acceptable since the time-dependence of $n_{L}$ would modify the equations of motion of the scalar field $\Phi$. Since $\dot{\tilde{\Phi}}=\dot{n}_{L} \Phi$, the above problem is resolved only if

$$
\frac{\dot{\tilde{\Phi}}}{\tilde{\Phi}}=\frac{\dot{n}_{L}}{n_{L}} \ll 1
$$

Moreover, any significant time evolution of the conformal factor $n_{L}$ would lead to the timedependence of either the fundamental mass scale, $m$, or the effective one, $\tilde{m}$.

It is straightforward to check that the above condition is indeed satisfied in our case. The expression for the lapse function at $y=L$ can be obtained using the relation $n_{L}(t)=$ $\lambda(t) \dot{a}_{L}(t)$ and eq. (3.22), and is found to be

$$
n_{L}(t)=\frac{a_{0}}{a_{L}} \frac{2 \Lambda_{R S}-\left(2 \Lambda_{1}-3 p_{1}-\rho_{1}\right) \tanh \left(\frac{A L}{2}\right)}{2 \Lambda_{R S}-\left(2 \Lambda_{2}-3 p_{2}-\rho_{2}\right) \tanh \left(\frac{A L}{2}\right)} .
$$

Differentiating the above expression with respect to time, we find

$$
\frac{\dot{n}_{L}}{n_{L}}=\frac{\dot{a}_{0}}{a_{0}}-\frac{\dot{a}_{L}}{a_{L}}+\frac{\left(3 w_{1}+1\right) \dot{\rho}_{1} \tanh \left(\frac{A L}{2}\right)}{2 \Lambda_{R S}\left[1-\alpha \tanh \left(\frac{A L}{2}\right)\right]}-\frac{\left(3 w_{2}+1\right) \dot{\rho}_{2} \tanh \left(\frac{A L}{2}\right)}{2 \Lambda_{R S}\left[1-\beta \tanh \left(\frac{A L}{2}\right)\right]},
$$

where we have set $p_{i}=w_{i} \rho_{i}$ and ignored higher-order terms of order $\mathcal{O}\left(\rho_{i} \dot{\rho}_{i}\right)$. As we have shown in the previous section, the first two terms are identical if we ignore corrections of order $\mathcal{O}\left(\rho_{i} / \Lambda_{R S}\right)^{2}$, which are extremely small if we recall that $\rho_{i} / \Lambda_{R S} \sim 10^{-123}$. The remaining two terms can be easily shown to be proportional to $\dot{\rho}_{i} / \Lambda_{R S} \sim\left(\rho_{i} / \Lambda_{R S}\right)^{3 / 2} M_{P}$ which undoubtly proves the validity of the condition (4.7). It may also be worth checking the validity of this argument at the time of the Big-Bang Nucleosynthesis. The corresponding term in the expression of $\dot{n}_{L}$ would be $10^{-148} M_{P}\left(\rho_{N} / \rho_{c}\right)^{3 / 2}$, where $\rho_{N}$ is the energy density of the universe during the BBN. If we assume that $T_{B B N} \simeq 10 \mathrm{MeV}$, we may easily find that $\rho_{N} / \rho_{c} \simeq 10^{39}$. This, in turn, leads to the final result that the magnitude of the term $\dot{\rho}_{i} / \Lambda_{R S}$ at the same period was of the order of $10^{-90} M_{P}$ rendering the time-dependence of the lapse function $n_{L}$ completely unobservable even in the early universe.

If we assume that the fundamental energy scale of the $5 \mathrm{D}$ theory in eq. (4.6), $m$, is of order $M_{P}$, while $\tilde{m} \sim 1 \mathrm{TeV}$, the rescaling coefficient should be of order $\sim 10^{-16}$. Expanding 
the result for the lapse function (4.8) and keeping only terms linear in $\rho_{i} / \Lambda_{R S}$, we obtain

$$
n_{L}=\sqrt{\frac{1-\alpha \tanh \left(\frac{A L}{2}\right)}{1-\beta \tanh \left(\frac{A L}{2}\right)}}\left\{1+\frac{\left(3 w_{1}+2\right) \rho_{1} \tanh \left(\frac{A L}{2}\right)}{2 \Lambda_{R S}\left[1-\alpha \tanh \left(\frac{A L}{2}\right)\right]}-\frac{\left(3 w_{2}+2\right) \rho_{2} \tanh \left(\frac{A L}{2}\right)}{2 \Lambda_{R S}\left[1-\beta \tanh \left(\frac{A L}{2}\right)\right]}\right\} .
$$

Since, the last two terms inside the brackets are negligible compared to unity, the condition that $n_{L} \simeq 10^{-16}$ should be imposed on the prefactor appearing in (4.10) involving $\alpha, \beta$ and $L$. Note that this prefactor is nothing more than the ratio of the scale factors on the two branes (3.21), once the small energy density contributions are ignored. By making use of eq. (3.23), this condition can be conveniently rewritten as

$$
n_{L}^{2}=\frac{1}{\cosh (A L)-\beta \sinh (A L)} \simeq 10^{-32},
$$

Once eq. (4.11) is imposed, we are left with only one independent parameter in the theory which we choose to be $L$, the inter-brane distance. The parameter $\beta$ can be determined by the condition (4.11), which together with the tuning of the cosmological constant (3.23) give the following expressions for $\alpha$ and $\beta$ in terms of $L$

$$
\alpha=\frac{\cosh (A L)-10^{-32}}{\sinh (A L)},
$$

and

$$
\beta=\frac{\cosh (A L)-10^{32}}{\sinh (A L)}
$$

According to our notation,

$$
A L=\sqrt{\frac{2}{3} \frac{\left|\Lambda_{B}\right|}{M_{5}^{3}}} L \simeq M_{P} L
$$

if we assume that $\left|\Lambda_{B}\right|^{1 / 5} \simeq M_{5} \simeq M_{P}$. In that case, the quantity $A L$ is nothing more than the distance between the two branes measured in units $M_{P}^{-1}$. Since the inter-brane distance is the free parameter of the theory, it would be interesting to see how the dependent parameters $\alpha$ and $\beta$ change as we vary $A L$. Their values will be dictated by eqs. (4.12) and (4.13), respectively, if we demand that both of the cosmological and the hierarchy problems are simultaneously solved. Numerical results for $\alpha$ and $\beta$ are plotted in Figure 1 as a function of $A L$ and some specific numerical examples are given in Table I.

Table I : The parameters $\beta$ and $1-\alpha$ for various values of the inter-brane distance $L$.

\begin{tabular}{|c|c|c|c|c|}
\hline \hline $\mathbf{A L}$ & $\mathbf{1}$ & $\mathbf{2 0}$ & $\mathbf{4 0}$ & $\mathbf{6 0}$ \\
\hline$\beta$ & $-8.5092 \times 10^{31}$ & $-9.0799 \times 10^{27}$ & $-8.4967 \times 10^{14}$ & $-1.7513 \times 10^{6}$ \\
\hline $1-\alpha$ & -0.31304 & $-4.1223 \times 10^{-9}$ & $-3.6097 \times 10^{-35}$ & $-1.5335 \times 10^{-52}$ \\
\hline \hline $\mathbf{A L}$ & $\mathbf{7 0}$ & $\mathbf{7 5}$ & $\mathbf{8 0}$ & $\mathbf{8 5}$ \\
\hline$\beta$ & -78.509 & 0.46427 & 0.99639 & 0.99998 \\
\hline $1-\alpha$ & $-3.0814 \times 10^{-61}$ & $3.9223 \times 10^{-65}$ & $3.6032 \times 10^{-67}$ & $1.6388 \times 10^{-71}$ \\
\hline \hline
\end{tabular}




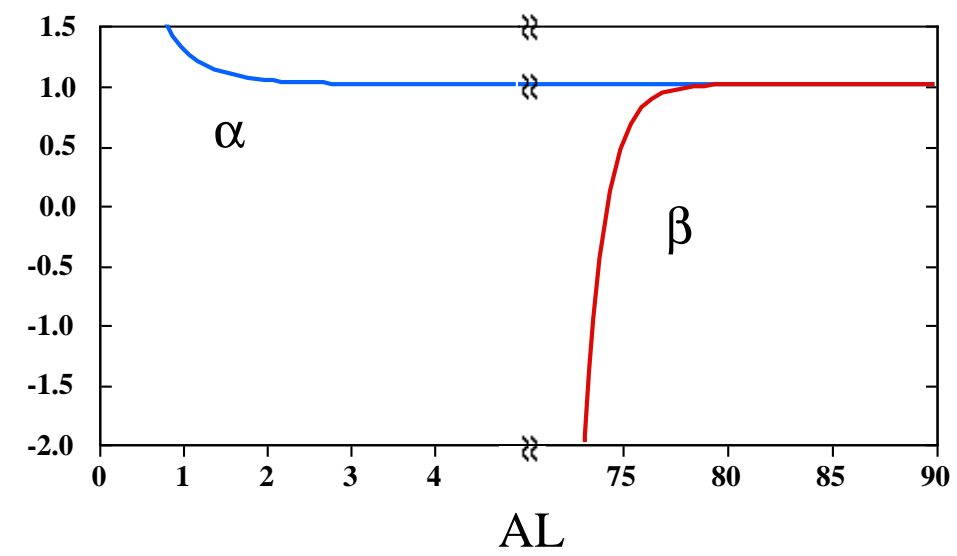

Figure 1: Behavior of the tensions (in units of $\Lambda_{R S}$ ), $\alpha$ and $\beta$ as a function of the interbrane separation (in units of $A^{-1}$ ).

We note that, for small values of the free parameter $L$, the brane parameter $\alpha$ is positive and of $\mathcal{O}(1)$ while $\beta$ is forced to acquire a value which is, not only negative, but also many orders of magnitude larger than that of $\alpha$. Such a situation is rather unphysical as it leads to an observable brane-universe with negative, total energy density and to an unexplained hierarchy between the sizes of the brane tensions. As $L$ increases, however, both $\alpha$ and $\beta$ start approaching unity: the combination $1-\alpha$ becomes extremely small while $\beta$ is moving towards less negative values. At $L \simeq 73.682722 \ldots$, the critical point $\beta=-1$ and $\alpha \simeq 1$, the choice of brane parameters in the Randall-Sundrum' model [3], is reached. If we further increase $L$, the combination $1-\alpha$ changes sign but it keeps approaching zero. On the other hand, $\beta$ becomes positive at a slightly larger value of the inter-brane distance, at $A L \simeq 75$. Any further increase in the value of $A L$ forces both of the brane parameters, $\alpha$ and $\beta$, to unity, which is the largest value that these parameters may take on. This is clear from eq. (3.23), for $\alpha=\beta=1$, where the hyperbolic tangent takes its asymptotic value, i.e. $\tanh (A L)=1$, and corresponds to an infinite distance between the two branes. Once the two branes become isolated, the only solution to the cosmological constant problem follows for $\alpha=\beta=1$. This is obvious from eq. (3.20), which in the limit $\tanh (A L) \rightarrow 1$, takes the form

$$
\left(\frac{\dot{a}_{0}}{a_{0}}\right)^{2}=\frac{\hat{\kappa}^{2}\left|\Lambda_{B}\right|}{3}\left[-1+\frac{\left(\rho_{1}+\Lambda_{1}\right)}{\Lambda_{R S}}\right] .
$$

In this limit, the expansion of the brane at $y=0$ becomes independent of the presence of the second brane at $y=L$ and the flatness of the corresponding $4 \mathrm{D}$ spacetime is guaranteed

\footnotetext{
${ }^{1}$ Note, that our conventions differ from those in Ref. [3] by a factor of 2 . Thus, their warp factor $e^{-k r_{c} \pi}$ corresponds to $e^{-A L / 2}$, in our case, if we ignore the matter on the brane and set $\alpha=-\beta=1$.
} 
only for $\alpha=1$ (similar results hold for the observable universe at $y=L$ ). Thus, for any value of the inter-brane distance, larger than, approximately, $75 M_{P}^{-1}$, the brane parameters are always positive, with their upper limit being unity, a result which leads to two braneuniverses with positive, total energy densities. Clearly, if $A L \gg 75$, then, in addition to tuning $1-\alpha$ to zero, $\beta$ must also be tuned to unity. We should stress here, once again, that all of the above values of $\alpha$ and $\beta$, either positive or negative, are in accordance with both the vanishing of the effective cosmological constant on both branes and with the resolution of the hierarchy problem on the observable universe.

Let us finally address the problem of the definition of the energy density, $\tilde{\rho}_{2}$, on our brane as measured by an observer living in a FRW spacetime in terms of the original energy density, $\rho_{2}$, defined in a $4 \mathrm{D}$ sub-space with lapse function $n_{L} \neq 1$. The energy-momentum tensor associated with the matter content of our universe is defined as

$$
T_{\mu \nu}^{M}=\frac{2}{\sqrt{g_{L}}} \frac{\delta S^{M}}{\delta g_{L}^{\mu \nu}},
$$

where $S_{M}$ is the part of the original action (2.2) defined only on our brane and containing time-dependent functions that describe the distribution of matter sources in our universe. After the conformal transformation (4.3), $T_{\mu \nu}^{M}$ changes as [17]

$$
T_{\mu \nu}^{M} \rightarrow \tilde{T}_{\mu \nu}^{M}=\frac{2}{\sqrt{\tilde{g}}} \frac{\delta S^{M}}{\delta \tilde{g}^{\mu \nu}}=n_{L}^{2} T_{\mu \nu}^{M}
$$

By using the fact that $\tilde{T}_{\nu}^{\mu}=\left(-\tilde{\rho}_{2}, \tilde{p}_{2}, \tilde{p}_{2}, \tilde{p}_{2}\right)$ and $\tilde{T}_{\nu}^{\mu}=\tilde{g}^{\mu \rho} \tilde{T}_{\rho \nu}=n_{L}^{4} T_{\nu}^{\mu}$, we finally obtain the results

$$
\tilde{\rho}_{2}=n_{L}^{4} \rho_{2}, \quad \tilde{p}_{2}=n_{L}^{4} p_{2} .
$$

The above definition of the observed energy density $\tilde{\rho}_{2}$ is in perfect agreement with the form of the Friedmann equation (3.26) for the cosmological expansion of our brane-universe. Recall that, to linear order in $\rho_{i}$, the expansion rate is

$$
\left(\frac{\dot{a}_{0}}{a_{0}}\right)^{2} \propto\left(\rho_{1}+n_{L}^{4} \rho_{2}\right)=\left(\rho_{1}+\tilde{\rho}_{2}\right)
$$

when one uses the expression for $n_{L}$ (4.11) in terms of the brane parameter $\beta$ and the inter-brane distance $A L$ (here, we consider only the lowest-order expression of $n_{L}$ since any $\rho_{i}$-dependent terms would create higher-order corrections, of $\mathcal{O}\left(\rho_{i}^{2}, \rho_{i} \rho_{j}\right)$, in the Friedmann equation). Note that no redefinition is necessary for the components of the $4 \mathrm{D}$ energymomentum tensor on the brane at $y=0$ since the lapse function, and thus the conformal factor, $n_{0}$, is unity. Similarly, we have $\tilde{a}_{0}=a_{0}$, and, as a result, eq. (4.19) still governs the expansion of the $4 \mathrm{D}$ FRW spacetime at $y=0$. Although the rescaling of the scale factor at $y=L$ is not trivial, we can easily see that

$$
\frac{\dot{\tilde{a}}_{L}}{\tilde{a}_{L}}=\frac{\dot{a}_{L}}{a_{L}}-\frac{\dot{n}_{L}}{n_{L}}=\frac{\dot{a}_{0}}{a_{0}}+\mathcal{O}\left(\frac{\rho_{i}^{2}}{\Lambda_{R S}^{2}}, \frac{\rho_{i} \rho_{j}}{\Lambda_{R S}^{2}}\right) .
$$


Therefore, in the linear-order approximation in the energy densities, the same equation

(3.26) describes the expansion of the 4D FRW spacetime at $y=L$. After performing the redefinition (4.18), the unique Friedmann equation (4.19) reveals the fact that the cosmological evolution of both branes is governed by the sum of the energy densities of the two branes as measured by observers living in flat, FRW 4D spacetimes. The same conclusion regarding the Friedmann expansion of both branes was also derived in Ref. [14] in the special case of the RS choice of the brane parameters $\alpha$ and $\beta$.

\section{Conclusions}

Before summarizing the work in this paper, let us emphasize briefly the main features of the two-brane models which may naturally explain the large ratio $\sim M_{P} / M_{W}$ via the hierarchy of the two length scales on different branes. In the RS model [3], the solution for the warp factor in the bulk, $a(y)$, was given by an exponential function of $y$ that interpolated between a positive and a negative self-energy brane. In the two-positive-brane model [[1], the corresponding solution behaved like a cosh-function characterized by the existence of a minimum. The solution to the hierarchy problem may be explained by the position of these two positive branes with respect to the minimum. Indeed, as we have shown, the ratio of the two lapse functions can be large, when the branes are located at non-equal distances from the minimum.

In this work, we have generalized these static models by introducing energy densities on the branes. Moreover, we have allowed for the possibility of arbitrary (positive or negative) brane tensions. We have shown that models of this type exhibit "normal" cosmology after demanding the vanishing of the effective cosmological constant on both branes and redefining the energy density on the observable brane in order to restore the FRW character of our $4 \mathrm{D}$ universe. It is also worth noting that no correlation between states of matter on the respective branes is required. In addition, we have shown that despite the timedependence of the lapse function on the observable brane, that defines the ratio $M_{P} / M_{W}$, its time evolution does not lead to any measurable change of the effective four-dimensional parameters since such changes would be proportional to $\rho_{i} / M_{P}^{4}$.

A crucial element in our model is the mechanism, responsible for the stabilization of the inter-brane distance. Here we simply assume that this mechanism exists without specifying physical causes, leading to the radion/dilaton stabilization. An extended discussion of this issue can be found in Ref. [10]. Of course, any model with a string like dilaton must ensure such a stabilization, since the dilaton expectation value fixes the gauge coupling and mass scales in the standard model.

We can not claim that two-brane models of this type are devoid of fine-tunings. As in all 
other known models, the cosmological constant requires a fine-tuning of different parameters in the model to ensure $\Lambda_{e f f}=0$. This reduces the initial set of three parameters (the two brane tensions relative to the bulk cosmological constant, and the inter-brane separation) to two. Note that the bulk cosmological constant relates the 4D and 5D (fundamental) Planck scales. When $\Lambda_{\text {eff }}$ is set to zero, the weak scale/Plank scale hierarchy problem may be explained by fixing a relation between the remaining brane tension and the inter-brane separation. For every value of the inter-brane distance, a solution to both the cosmological constant problems and the hierarchy problem can be derived by appropriately choosing the tension of the observable universe. While for small values of $A L$, the brane parameter $\beta$ is forced to acquire a large negative value, as the inter-brane distance increases, both of the parameters $\alpha$ and $\beta$ start decreasing (in absolute value) towards unity. The RS choice of parameters, i.e. $\alpha=-\beta=1$, arises as a special solution when we reach the value $A L \simeq 73$ while, for all separations $A L \gtrsim 75$, more natural solutions (with positive brane tensions for both branes) arise. The parameters $\alpha$ and $\beta$ remain positive no matter how large the inter-brane separation becomes. The asymptotic value $\alpha=\beta=1$ is reached in the limit of infinite $A L$, when the two branes become isolated. In this limit, the problem of the cosmological constant is solved by imposing independent conditions on each brane and the framework for the resolution of the hierarchy problem ceases to exist.

Acknowledgments This work was supported in part by the Department of Energy under Grant No. DE-FG-02-94-ER-40823 at the University of Minnesota.

\section{References}

[1] E. Witten, Nucl. Phys. B471 (1996) 135; P. Horava, and E. Witten, Nucl. Phys. B460 (1996) 506; B475 (1996) 94; T. Banks and M. Dine, Nucl. Phys. B479 (1996) 173.

[2] N. Arkani-Hamed, S. Dimopoulos and G. Dvali, Phys. Lett. B429 (1998) 263;

I. Antoniadis, N. Arkani-Hamed, S. Dimopoulos and G. Dvali, Phys. Lett. B436 (1998) 257.

[3] L. Randall and R. Sundrum, Phys. Rev. Lett. 83 (1999) 3370.

[4] A.G. Cohen and D.B. Kaplan, Phys. Lett. B470 (1999) 52; J. Lykken and L. Randall, JHEP 0006 (2000) 014; Z. Chacko and A.E. Nelson, hep-th/9912186; M. Chaichian and A.B. Kobakhidze, Phys. Lett. B478 (2000) 299; N. Arkani-Hamed, L. Hall, D. Smith, and N. Weiner, hep-ph/9912453.

[5] A. Lukas, B.A. Ovrut, K.S. Stelle and D. Waldram, Phys. Rev. D59 (1999) 086001; A. Lukas, B.A. Ovrut and D. Waldram, Phys. Rev. D60 (1999) 086001; Phys. Rev. D61 (2000) 023506. 
[6] N. Kaloper and A. Linde, Phys. Rev. D59 (1999) 101303; N. Kaloper, Phys. Rev. D60 (1999) 123506.

[7] P. Binétruy, C. Deffayet and D. Langlois, Nucl. Phys. B565 (2000) 269; E. Flanagan, S.H.H. Tye and I. Wasserman, Phys. Rev. D62 (2000) 044039.

[8] P. Kanti, I. Kogan, K.A. Olive and M. Pospelov, Phys. Lett. B468 (1999) 31.

[9] C. Csáki, M. Graesser, L. Randall, and J. Terning, Phys. Rev. D62 (2000) 045015.

[10] P. Kanti, I. Kogan, K.A. Olive and M. Pospelov, Phys. Rev. D61 (2000) 106004.

[11] P. Kanti, K.A. Olive and M. Pospelov, Phys. Lett. B481 (2000) 386.

[12] C. Csáki, M. Graesser, C. Kolda and J. Terning, Phys. Lett. B462 (1999) 34; J.M. Cline, C. Grojean and G. Servant, Phys. Rev. Lett. 83 (1999) 4245; D.J.H. Chung and K. Freese, Phys. Rev. D61 (2000) 023511; H.B. Kim and H.D. Kim, Phys. Rev. D61 (2000) 064003; P. Binétruy, C. Deffayet, U. Ellwanger and D. Langlois, Phys. Lett. B477 (2000) 285; N. Arkani-Hamed, S. Dimopoulos, G. Dvali, and N. Kaloper, hep-ph/9911386; D. Ida, gr-qc/9912002; N. Kaloper, Phys. Lett. B474 (2000) 269; I.I. Kogan, S. Mouslopoulos, A. Papazoglou, G.G. Ross, and J. Santiago, hep-ph/9912552; U. Elwanger, hep-th/0001126; I.I. Kogan, and G.G. Ross, Phys. Lett. B485 (2000) 255; J.E. Kim, B. Kyae, and H.M. Lee, hep-th/0004005; C. Barcelo and M. Visser, Phys. Lett. B482 (2000) 183; H. Stoica, S.-H. Tye, and I. Wasserman, Phys. Lett. B482 (2000) 205; B. Grinstein, D.R. Nolte, and W. Skiba, hep-th/0005001; M. Brändle, A. Lukas and B.A. Ovrut, hep-th/0003256.

[13] H.B. Kim, Phys. Lett. B478 (2000) 285.

[14] J. Lesgourgues, S. Pastor, M. Peloso and L. Sorbo, hep-ph/0004086.

[15] R.N. Mohapatra, A. Pérez-Lorenzana and C.A. de S. Pires, hep-ph/0003328.

[16] U. Ellwanger, Phys. Lett. B473 (2000) 233.

[17] C. Wetterich, Nucl. Phys. B302 (1988) 645. 